Case Report

\title{
Acute Monocytic Leukemia Masquerading Behçet's Disease-Like Illness at Onset in an Elderly Female
}

\author{
Shigeru Koba, ${ }_{1}^{1}$ Toshio Sekioka, ${ }^{1}$ Sorou Takeda, ${ }^{1}$ Aya Miyagawa-Hayashino, ${ }^{2}$ \\ Keisuke Nishimura, ${ }^{3}$ and Shinsaku Imashuku ${ }^{4}$ \\ ${ }^{1}$ Department of Internal Medicine, Uji-Tokushukai Medical Center, Uji 611-0042, Japan \\ ${ }^{2}$ Department of Diagnostic Pathology, Kyoto University Hospital, Kyoto 606-8507, Japan \\ ${ }^{3}$ Division of Pathology, Uji-Tokushukai Medical Center, Uji 611-0042, Japan \\ ${ }^{4}$ Department of Laboratory Medicine, Uji-Tokushukai Medical Center, Uji 611-0042, Japan
}

Correspondence should be addressed to Shinsaku Imashuku; shinim95@mbox.kyoto-inet.or.jp

Received 31 May 2016; Accepted 25 July 2016

Academic Editor: Kazunori Nakase

Copyright (C) 2016 Shigeru Koba et al. This is an open access article distributed under the Creative Commons Attribution License, which permits unrestricted use, distribution, and reproduction in any medium, provided the original work is properly cited.

\begin{abstract}
A previously healthy 74-year-old Japanese female was hospitalized with fever and high C-reactive protein. She developed palatal herpangina-like aphthous ulcers, localized intestinal wall thickening, terminal ileum ulcers, and an erythematous acneiform rash; thus Behçet's disease-like illness was suspected. Significant peripheral blood acute monocytosis developed during her hospitalization and acute monocytic leukemia (FAB M5b) with normal karyotype was diagnosed. By immunostaining, the infiltrating cells in the skin and the terminal ileum were identified as monocytic leukemic cells. This case exhibited a unique initial presentation of Behçet's disease-like illness associated with acute monocytic leukemia.
\end{abstract}

\section{Introduction}

Autoimmune diseases like Behçet's disease (BD) develop in as many as $10 \%$ of patients with myelodysplastic syndromes (MDS) or myeloproliferative neoplasms (MPN) [1]. In particular, a link between $\mathrm{BD}$ or BD-like illness and chronic myelomonocytic leukemia (CMML) with a trisomy 8 chromosome abnormality has been well recognized [2-4]. However, the association of $\mathrm{BD}$ or BD-like illness and acute myeloid leukemia has rarely been reported [5]. In patients with CMML showing cutaneous lesions as a sign of BD or BD-like illness, skin tissues were shown to contain abnormal monocytes, that is, leukemia cutis [6-8]. We report here an elderly Japanese female who initially presented with BDlike illness including cutaneous lesions and thereafter was diagnosed as having acute monocytic leukemia (AMoL; FAB M5b). In this case, we identified leukemic cells in the tissues of skin and ileum.

\section{Case Report}

A previously healthy 74-year-old Japanese female was hospitalized with unknown fever and high C-reactive protein
(CRP) values in December 2015. On admission, she was $155 \mathrm{~cm}$ in height and $59.2 \mathrm{~kg}$ in weight and had temperature of $37.6^{\circ} \mathrm{C}$, blood pressure of $118 / 64 \mathrm{mmHg}$, pulse rate of $71 / \mathrm{min}$, respiration rate of $18 / \mathrm{min}$, and $\mathrm{SpO}_{2}$ of $94 \%$ at room temperature. Physical examination revealed no specific findings. After admission, she developed herpangina-like aphthous ulcers at the palate, when significant monocytosis (absolute monocyte counts $>5,000 / \mu \mathrm{L}$ ) was first noted; however, monocytosis fluctuated (Figure 1). Thereafter, a CT scan of the abdomen showed localized thickening of the intestinal wall (Figure 2(a)) and colonofiberscopy (CF) revealed the presence of multiple ovoid punched-out ulcers at the terminal ileum and aphthous lesions at the ascending colon (Figure 2(b)). In addition, the patient also developed multiple erythematous rashes on her right thigh (Figure 3(a)). Given the diagnostic criteria of BD [9], the rash was not typical erythema nodosum but was thought rather to be acneiform eruption. Ophthalmological studies showed no evidence of BD signs. Taken together, BD was suspected in this patient from fulfilling 2 major and 1 minor clinical features required in the diagnosis of BD [9]. Thereafter, from the second week of admission, the patient again developed 


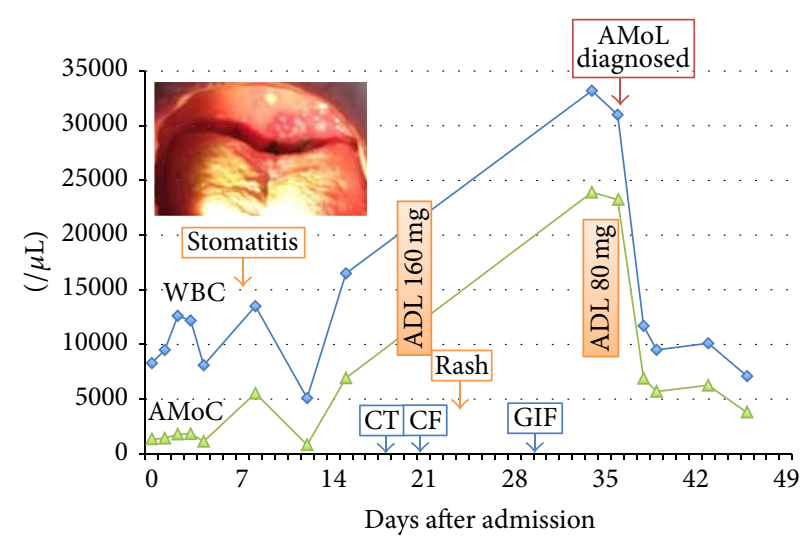

FIGURE 1: Clinical course of the patient: the patient first developed aphthous stomatitis (inserted photo), followed by intestinal BD-like lesions detected by CF and then skin rash. Significant monocytosis triggered by urinary tract infection was noted at the 5th week of admission and the eventual diagnosis of AMoL was made. WBC: white cell counts, AMoC: absolute monocyte counts, ADL: adalimumab, CT: computerized tomography, CF: colonofiberscopy, GIF = gastrointestinal fiberscopy.

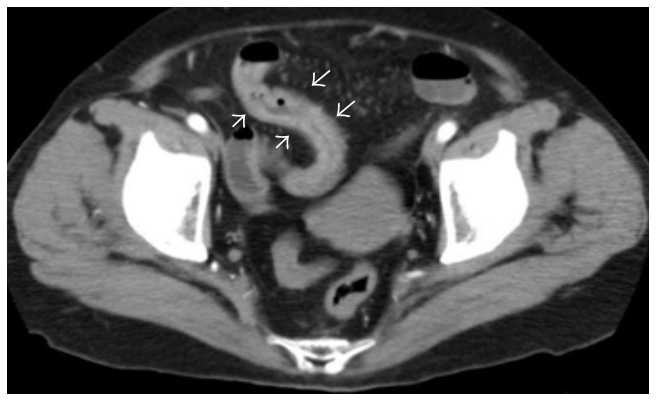

(a)

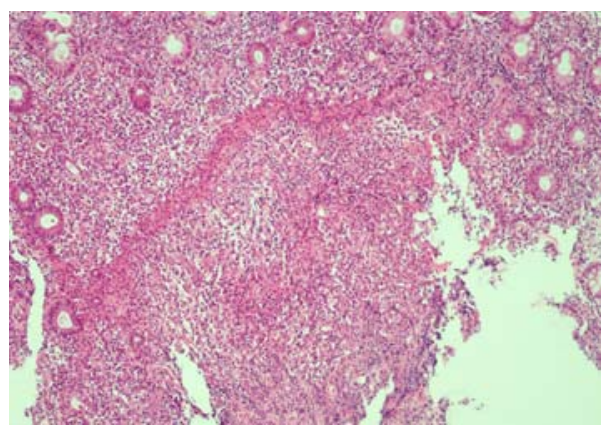

(c)

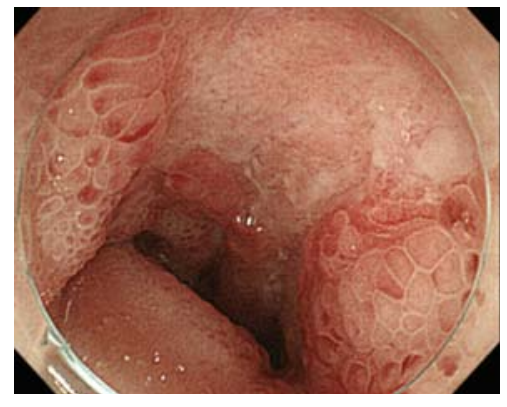

(b)

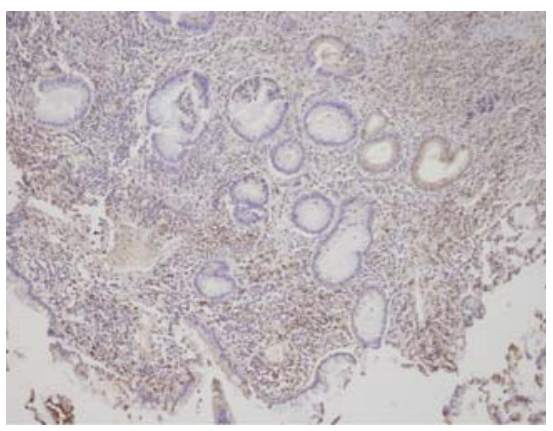

(d)

FIGURE 2: Intestinal findings. Abdominal CT scan showed localized thickening of the intestinal wall (arrows) (a). Endoscopic study revealed multiple ovoid punched-out ulcers at the terminal ileum (b). HE staining of a biopsy of the terminal ileum showed the presence of ulcerassociated granulation tissues with vascularization and infiltration of cells (original magnification $\times 100)(\mathrm{c})$, which were strongly positive for lysozyme (d) (original magnification $\times 100$ ). Positive stains for CD13, CD14, and CD33 are not shown.

a significant monocytosis (from $15 \%$ to $>70 \%$ in $\mathrm{PB}$, with absolute monocyte counts to a maximum of $23,900 / \mu \mathrm{L}$ ) associated with urinary infection at the 5 th week of admission (Figure 1). These findings with subsequent bone marrow study confirmed a diagnosis of AMoL (Figures 4(a) and 4(b)).

Blood results at the 5 th week of admission, when AMoL was diagnosed, were as follows: white blood cells
$(33,200 / \mu \mathrm{L})$ containing $15 \%$ blasts, $21 \%$ promonocytes, $19 \%$ mature monocytes, $1 \%$ myelocytes, $10 \%$ neutrophils, and $33 \%$ lymphocytes (Figure 4(a)). Flow cytometric data of a major cell population in $\mathrm{PB}$ were as follows: CD13+ $(86.4 \%)$, $\mathrm{CD} 14+$ (80.0\%), CD33+ (97.4\%), CD34+ (0.8\%), CD41+ (44.8\%), CD56+ (32.4\%), and HLA-DR+ (97.0\%). Mature lymphocytes in PB consisted of mostly T cells (approximately 


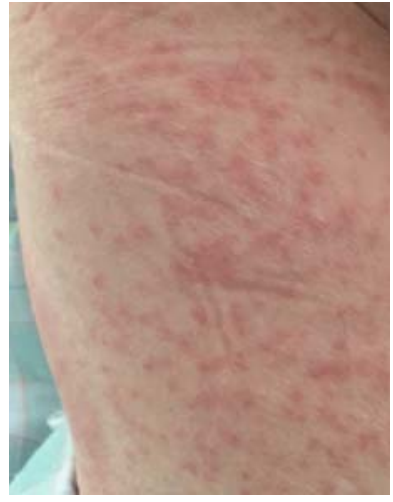

(a)

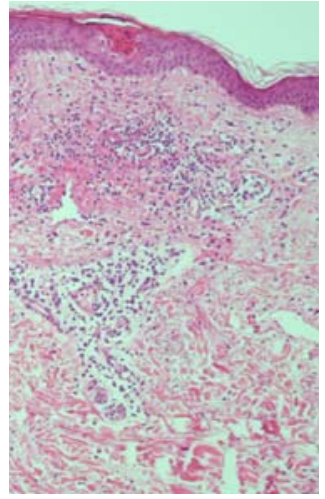

(b)

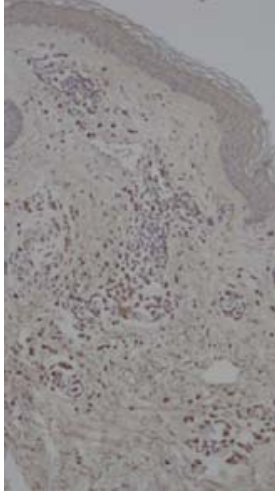

(c)

FIgURE 3: Findings of cutaneous lesions: photo of the rash on the right thigh (a). The biopsy shows the dense perivascular and periadnexal infiltration of mononuclear cells in the dermis extending into the subcutaneous tissue (b) (HE staining, original magnification $\times 100$ ). The mononuclear cells were strongly lysozyme-positive (c) (original magnification $\times 100$ ). Positive stains for CD13, CD14, and CD33 are not shown.

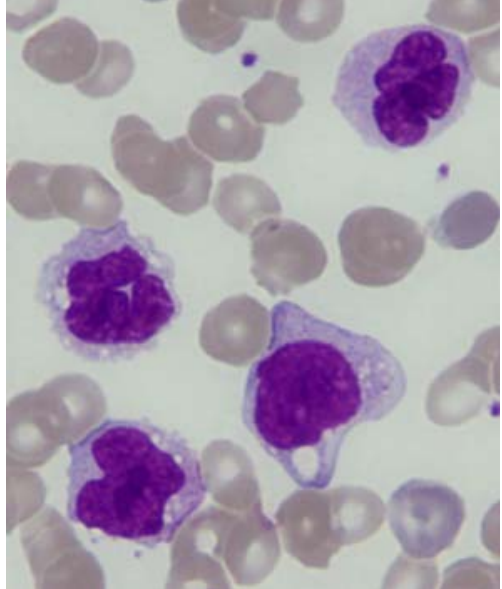

(a)

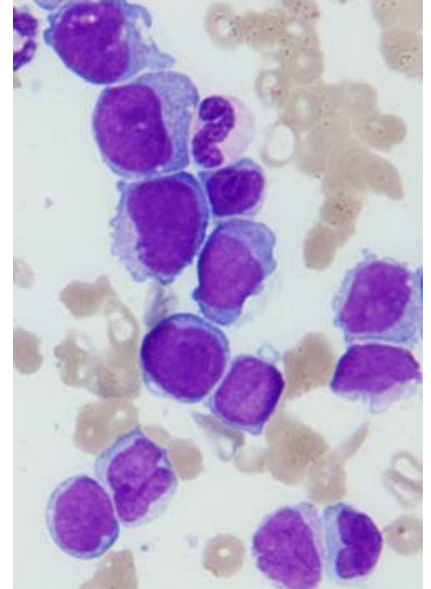

(b)

FIGURE 4: May-Grünwald-Giemsa stained smears of peripheral blood and bone marrow show mature monocytes and promonocytoid cells with folded nuclei in the peripheral blood (a) and immature blast and promonocytoid cells in the bone marrow (b) (original magnification $\times 1,000)$.

90\%), whose markers were CD4+ (59.2\%) and CD8+ (27.4\%), respectively. By contrast, the hypercellular BM consisted of $33.2 \%$ blasts, $15.0 \%$ promonocytes, $33.6 \%$ mature monocytes, $6.8 \%$ granulocytes, $1.4 \%$ lymphocytes, $2.0 \%$ plasma cells, and $8.0 \%$ erythroblasts with an M/E ratio of 0.85 (Figure $4(\mathrm{~b})$ ). No significant abnormalities indicating MDS were noted. Flow cytometric results of the major cell population in BM were as follows: CD13+ (38.3\%), CD14+ (26.0\%), CD33+ (97.5\%), CD $41+(45.5 \%), \mathrm{CD} 34+(2.3 \%), \mathrm{CD} 56+(38.0 \%)$, and HLADR+ $(80.9 \%)$. Mature lymphocytes in BM were mostly $\mathrm{T}$ cells (approximately 90\%) with a CD4/CD8 ratio of 0.54. Myeloperoxidase positive cells accounted for $10 \%$ of the mononuclear cells present in both the $\mathrm{PB}$ and $\mathrm{BM}$, while alpha-naphthyl butyrate esterase (inhibited by sodium fluoride) positive cells comprised more than $60 \%$ of cells in both the $\mathrm{PB}$ and $\mathrm{BM}$. These findings were compatible with the diagnostic criteria of AMoL (FAB M5b) [10]. In addition, the karyotypes of $\mathrm{PB}$ and $\mathrm{BM}$ cells were both 46, XX [20/20]. The other blood chemistry was uneventful except for high levels of CRP $(15.75 \mathrm{mg} / \mathrm{dL})$, with aspartate aminotransferase $13 \mathrm{U} / \mathrm{L}$, alanine aminotransferase $14 \mathrm{U} / \mathrm{L}$, lactate dehydrogenase $205 \mathrm{U} / \mathrm{L}$, total bilirubin $0.76 \mathrm{mg} / \mathrm{dL}$, total protein $6.9 \mathrm{~g} / \mathrm{dL}$, albumin $2.9 \mathrm{~g} / \mathrm{dL}$, blood urea nitrogen $23.4 \mathrm{mg} / \mathrm{dL}$, creatinine $1.61 \mathrm{mg} / \mathrm{dL}$, sodium $135 \mathrm{mmol} / \mathrm{L}$, potassium $3.6 \mathrm{mmol} / \mathrm{L}$, chlorine $97 \mathrm{mmol} / \mathrm{L}$, and calcium $8.0 \mathrm{mg} / \mathrm{dL}$.

Since the diagnosis of AMoL (FAB M5b) was confirmed, the biopsied tissues showing BD-like illness were reevaluated with immunostaining except for stomatitis lesions and were proved to have leukemic cell infiltrations. The intestinal ulcer-associated granulation tissues with vascularization and infiltration of cells were positive for lysozyme, CD13, CD14, 
and CD33 (Figures 2(c) and 2(d)). Also the histopathology of skin rash showed that the infiltrating cells were positive for lysozyme, CD13, CD14, and CD33 (Figures 3(b) and $3(c))$. These findings confirmed that the lesions primarily thought due to BD-like illness were in fact AMoL-related. In addition, HLA-typing, performed to ascertain whether the patient had BD-related HLA types, detected the presence of A2/A24 and B52/B55 but not the BD-related HLA-B51 or B5 alleles [11, 12]. As shown in Figure 1, the patient was initially treated with adalimumab for BD-like illness, following the successful report for a case of intestinal BD with trisomy 8 MDS by Kimura et al. [13]; however, after AMoL was confirmed, she was transferred to another hospital for intensive chemotherapy as a very high-risk patient.

\section{Discussion}

This case is not a classic autoimmune case of $\mathrm{BD}$, nor is $\mathrm{AMoL}$ that developed during the treatment for $\mathrm{BD}$. AMoL masquerading BD-like illness acutely developed. We had no evidence that this case progressed from CMML, because blood count records for the past 5 years prior to admission showed no increase of monocytes (which remained $<10 \%$ ) in the PB. Clinical features of BD, aphthous stomatitis, skin lesions, and ulcers in the terminal ileum were present, but genital ulcers, vascular, or ocular involvements were absent; thus, it was considered to be an incomplete $\mathrm{BD}$ or $\mathrm{BD}$-like illness. The HLA-B51 allele was also absent. To date, the association of $\mathrm{BD}$ or $\mathrm{BD}$-like illness with MDS has been well characterized, particularly in those patients with CMML $[2-4,14-16]$, but rarely characterized in patients with acute myeloid leukemia [5]. The precise causes developing $\mathrm{BD}$ or BD-like illness in cases of MDS/CMML are unknown [17].

In immunostaining studies in a classic autoimmune case of BD, Yamana et al. [18] showed that the lymphocytes infiltrating the terminal ileum were high $\mathrm{CD} 4\left(\right.$ Leu 3a) ${ }^{+}$cells and low CD8 (Leu 2a) ${ }^{+}$cells. On the other hand, Tada et al. [14] previously reviewed the clinical characteristics of MDSassociated BD or BD-like illness in Japan but did not mention the characteristics of infiltrating cells in the tissues. In CMML cases, leukemic cells were reported to infiltrate cutaneous tissues as leukemia cutis [6-8]. We confirmed that both the skin rash and ileal tissues were infiltrated with monocytic leukemic cells in the case presented here. It remains unknown however how these findings explain the development of $\mathrm{BD}$ or BD-like illness in a case of AMoL. In a classic autoimmune case of $\mathrm{BD}$ cytokine-producing dysfunctional $\mathrm{T}$ cells play a major role [19]. In our case, infiltrating monocytic leukemic cells or $\mathrm{T}$ cells responding to monocytic leukemic cells or both might have played a similar role by producing various cytokines. As another interesting issue, a literature survey indicates a correlation of $\mathrm{BD}$ or BD-like illness and MDS/CMML with the presence of chromosomal trisomy 8 $[2-4,15,16]$. The present case, in contrast, had no trisomy 8 .

In summary, although rare, caution must be exercised if $\mathrm{BD}$ or BD-like illness is associated with any hematological diseases, such as MDS/CMML, or rarely AMoL. We presented here the unique clinical course of an elderly patient whose disease initiated with the clinical features mimicking $\mathrm{BD}$ and was followed by the diagnosis of AMoL (FAB M5b) with normal karyotype.

\section{Competing Interests}

The authors declare no competing interests.

\section{Acknowledgments}

The authors thank Dr. Taro Sakamaki, Department of Hematology and Oncology, Graduate School of Medicine, Kyoto University, for his helpful discussion and advice on this case.

\section{References}

[1] M. Castro, D. L. Conn, W. P. D. Su, and J. P. Garton, "Rheumatic manifestations in myelodysplastic syndromes," The Journal of Rheumatology, vol. 18, no. 5, pp. 721-727, 1991.

[2] Y.-C. Lin, T.-H. Liang, H.-N. Chang, J.-S. Lin, and H.-Y. Lin, "Behçet disease associated with myelodysplastic syndrome," Journal of Clinical Rheumatology, vol. 14, no. 3, pp. 169-174, 2008.

[3] M. G. Mantzourani, K. Chantziara, I. Thanopoulou, H. Variami, G. Vaiopoulos, and G. A. Pangalis, "Coexistence of Behcet's disease and chronic myelomonocyte leukemia with trisomy 8: a case report and review of the literature," Clinical and Experimental Rheumatology, vol. 27, no. 2, pp. S85-S87, 2009.

[4] C. Muñoz-Grajales, F. Z. González, R. B. Arenas et al., "Behçet's disease, chronic myelomonocytic leukemia and trisomy 8," International Journal of Clinical Rheumatology, vol. 9, no. 2, pp. 115-120, 2014.

[5] A. Kaloterakis, N. G. Stavrianeas, I. N. Karagianni et al., "Adamantiades-Behcet's disease coexisting with acute myeloblastic leukaemia," British Journal of Dermatology, vol. 137, no. 2, pp. 317-318, 1997.

[6] H. Suzuki, T. Takahashi, T. Hayashi et al., "Rapidly progressed chronic myelomonocytic leukemia associated with severe skin infiltration," Rinsho Ketsueki, vol. 38, no. 9, pp. 752-756, 1997 (Japanese).

[7] L. L. Yavorkovsky, J. Zain, C. D. Wu, L. Trivelli, and P. Cook, "Monocytic leukemia cutis diagnosed simultaneously with refractory anemia with monocytosis: a case report," American Journal of Hematology, vol. 66, no. 2, pp. 120-122, 2001.

[8] R. A. Mathew, J. M. Bennett, J. J. Liu et al., "Cutaneous manifestations in CMML: indication of disease acceleration or transformation to AML and review of the literature," Leukemia Research, vol. 36, no. 1, pp. 72-80, 2012.

[9] M. S. Kurokawa and N. Suzuki, "Behcet's disease," Clinical and Experimental Medicine, vol. 4, no. 1, pp. 10-20, 2004.

[10] J. M. Bennett, D. Catovsky, M. T. Daniel et al., "Proposed revised criteria for the classification of acute myeloid leukemia. A report of the French-American-British Cooperative Group," Annals of Internal Medicine, vol. 103, no. 4, pp. 620-625, 1985.

[11] K. Sakly, R. Lahmar, F. Nefzi et al., "Phenotypic abnormalities of peripheral blood mononuclear cells in patients with Behçet's disease and association with HLA-B51 expression," Immunological Investigations, vol. 43, no. 5, pp. 463-478, 2014.

[12] C. Maldini, M. P. Lavalley, M. Cheminant, M. de Menthon, and A. Mahr, "Relationships of HLA-B51 or B5 genotype with 
Behçet's disease clinical characteristics: systematic review and meta-analyses of observational studies," Rheumatology, vol. 51, no. 5, Article ID ker428, pp. 887-900, 2012.

[13] M. Kimura, Y. Tsuji, M. Iwai et al., "Usefulness of adalimumab for treating a case of intestinal Behçet's disease with trisomy 8 myelodysplastic syndrome," Intestinal Research, vol. 13, no. 2, pp. 166-169, 2015.

[14] Y. Tada, S. Koarada, Y. Haruta, M. Mitamura, A. Ohta, and K. Nagasawa, "The association of Behçet's disease with myelodysplastic syndrome in Japan: a review of the literature," Clinical and Experimental Rheumatology, vol. 24, no. 5, supplement 42, pp. S115-S119, 2006.

[15] H. Ogawa, T. Kuroda, M. Inada et al., "Intestinal Behçet's disease associated with myelodysplastic syndrome with chromosomal trisomy 8-a report of two cases and a review of the literature," Hepato-Gastroenterology, vol. 48, no. 38, pp. 416-420, 2001.

[16] L. Eder, M. Rozenbaum, N. Boulman et al., "Behçet's disease, myelodysplastic syndrome, trisomy 8 , gastroenterological involvement-an association," Clinical and Experimental Rheumatology, vol. 23, no. 4, supplement 38, pp. S91-S95, 2005.

[17] T. Braun and P. Fenaux, "Myelodysplastic Syndromes (MDS) and autoimmune disorders (AD): cause or consequence?" Best Practice and Research: Clinical Haematology, vol. 26, no. 4, pp. 327-336, 2013.

[18] S. Yamana, S. L. Jones, T. Shimamoto et al., "Immunohistological analysis of lymphocytes infiltrating the terminal ileum in a patient with intestinal Behçet's disease," Ryumachi, vol. 26, no. 1, pp. 10-14, 1986 (Japanese).

[19] N. Sugi-Ikai, M. Nakazawa, S. Nakamura, S. Ohno, and M. Minami, "Increased frequencies of interleukin-2- and interferon- $\gamma$-producing T cells in patients with active Behcet's disease," Investigative Ophthalmology \& Visual Science, vol. 39, no. 6, pp. 996-1004, 1998. 


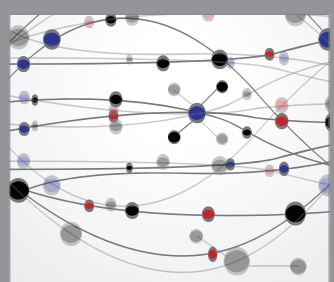

The Scientific World Journal
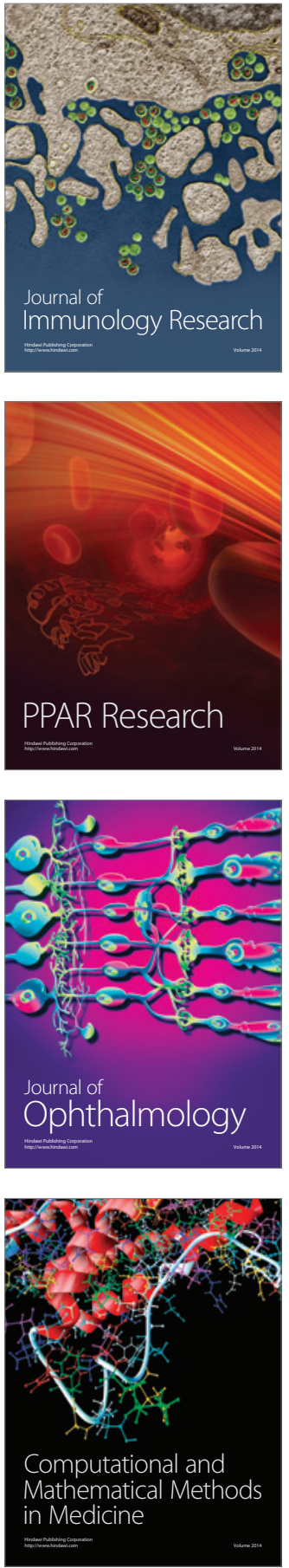

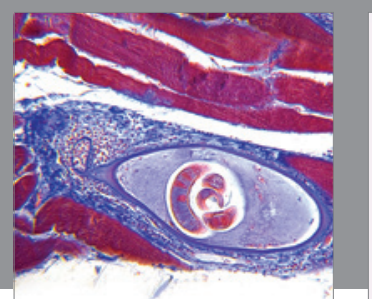

Gastroenterology Research and Practice

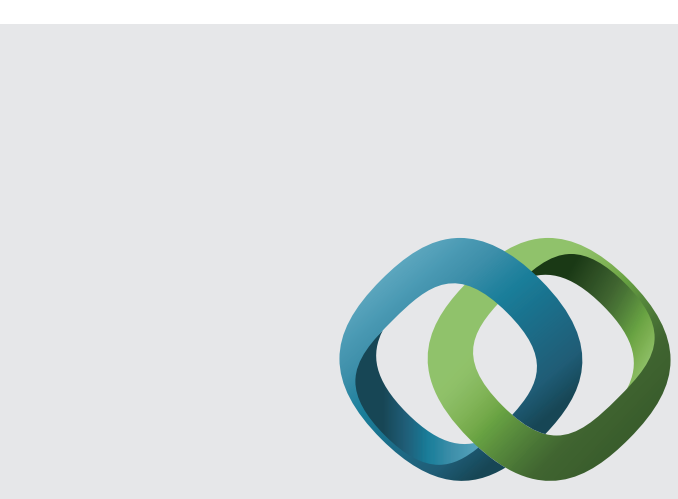

\section{Hindawi}

Submit your manuscripts at

http://www.hindawi.com
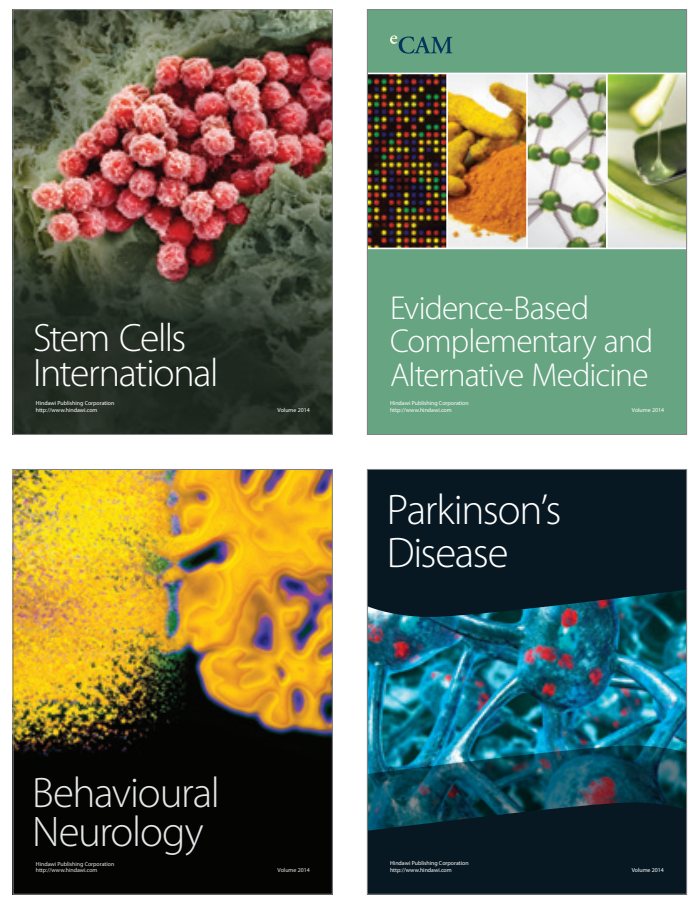
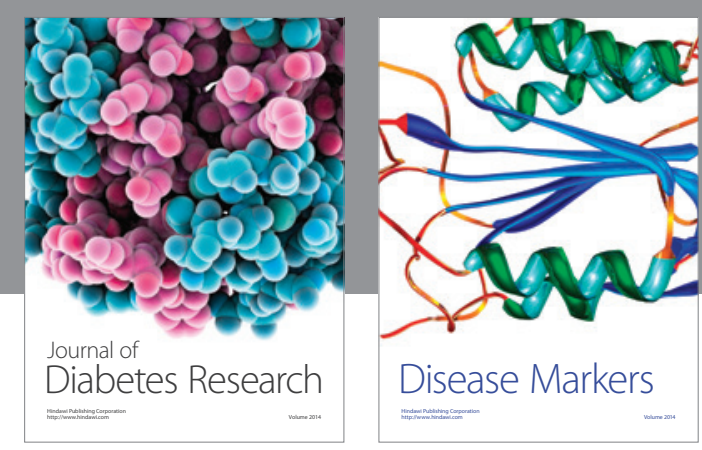

Disease Markers
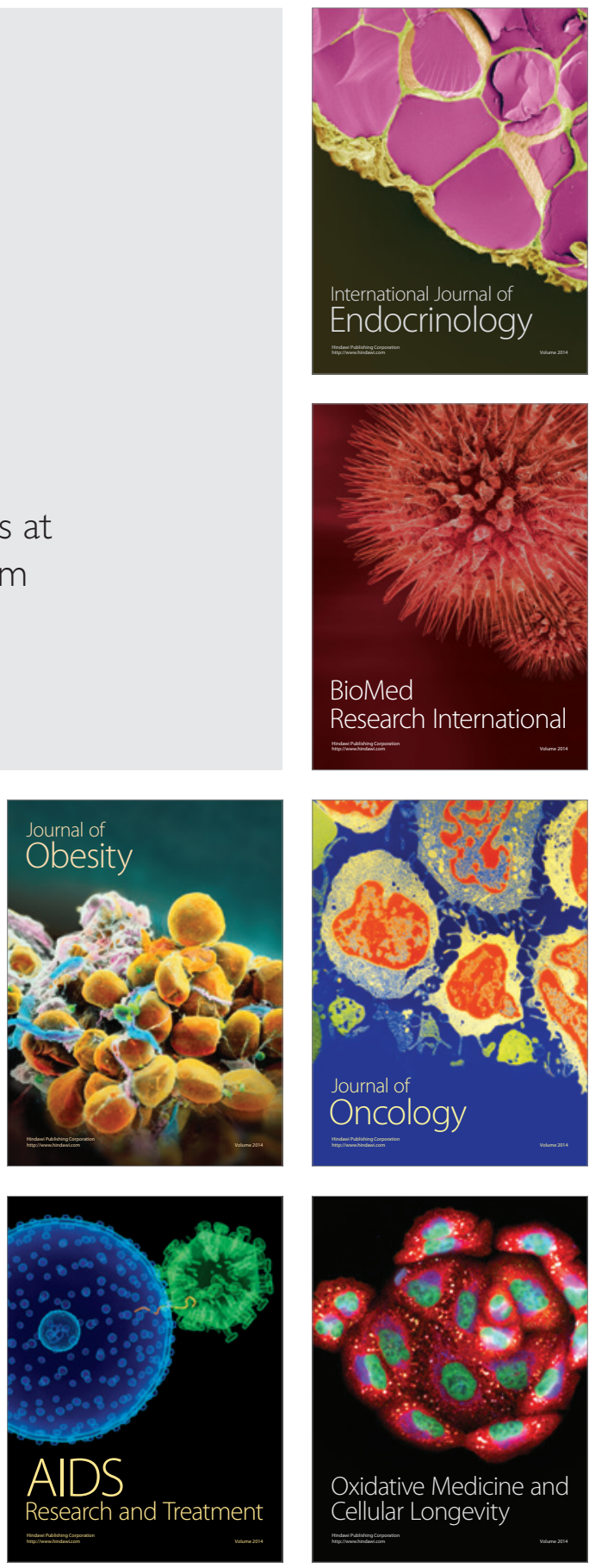